\title{
Nucleation Phenomena Interpreted by Fluctuation/Dissipation Theory
}

\author{
Nicholas F. Borrelli, Joseph F. Schroeder \\ Corning Inc. Research Lab. Sullivan Park, New York, USA \\ Email address: \\ Borrellinf@Corning.com (N. F. Borrelli), Schroedejf@Corning.com (J. F. Schroeder) \\ ${ }^{*}$ Corresponding author
}

\section{To cite this article:}

Nicholas F. Borrelli, Joseph F. Schroeder. Nucleation Phenomena Interpreted by Fluctuation/Dissipation Theory. American Journal of Modern Physics. Vol. 10, No. 3, 2021, pp. 51-54. doi: 10.11648/j.ajmp.20211003.12

Received: April 14, 2021; Accepted: May 7, 2021; Published: May 14, 2021

\begin{abstract}
An argument is presented in the attempt to link the nucleation phenomenon to a broader interpretation and manifestations termed "Fluctuation and Dissipation theory". The connection is made that the nucleation phenomenon can be thought of as being developed from "noise" which in the case of glass and crystals is spontaneous as the composition fluctuates. In many ways it will be shown it to have phenomenological similarities to turbulence and the Liesegang phenomenon, and perhaps even to some extent, evolution [1] which all having sudden threshold-like behaviors. In this paper an argument will be made of the common features of these three phenomena by comparing the mathematical equations describing the three phenomena and indicating that they inherently contain the terms that give rise to instability and how this can explained with recourse to fluctuation/dissipation theory.
\end{abstract}

Keywords: Nucleation, Fluctuation, Glass-ceramics

\section{Nucleation}

Nucleation is a phenomenon in solids where a new phase is produced from the otherwise homogeneous system using a thermal development schedule. This is conventionally broken down into two separate and sequential steps - the first termed "nucleation" and the other is called "growth". Although this use of separate terminology might be useful to describe the time sequence of the phenomenon in general it tends to gloss over what the respective terms mean in physical terms - in other words what might be the underlying phenomenon that occurs. For example, the nucleation stage, more often than not in glass-ceramics [2-6] is attributed to specific added elements to the system also referred to as "nucleating agents." For example, in glass-ceramic systems agents such as Titania [3] or Zirconia are added to initiate the nucleation. In other systems elements such as fluorine or even noble metals [7] are used but one must be careful to distinguish between phase separation (e.g. separate titanium-rich phase being formed) as opposed to nucleation as mechanism unto itself even though the former could lead to crystallization; that is to distinguish from the view that all nucleation derives from some sort of initial phase separation no matter how small or non-detectable it may be. This is the salient distinction between agents that enhance nucleation and the actual initial source.

In this paper, an attempt will be made to cast the nucleation phenomenon in a different way that is in terms of what is known in Physics as "fluctuation and dissipation theory". [8] This theory is used to study, for instance, among other such concepts as inherent noise in systems [9]. It is interesting to think as nucleation as resulting from "noise" or inherent spontaneous composition or structural fluctuations in the system. Perhaps, even more surprising is to consider an entirely different example such as a possible way to look at "turbulence" in fluid flow. [10] Here too, a spontaneous phenomenon in flow suddenly appears as the velocity increases to some critical value. Another phenomenon that might be related is the mechanism by which Liesegang layers spontaneous form in certain materials. These examples will be briefly mentioned below. It is not the intention to imply that these phenomena are physically related in any real way but rather to indicate they all share a common sudden onset of a property change that could be suggestive of a fluctuation-based origin.

Although certainly well beyond the intent of this paper it 
is, nonetheless, interesting to point out that there is a theory of the origin of life called "random statistical fluctuations [1] in contrast to Darwin's "natural selection". What this pertains to is the initial formation of RNA (nucleic acids) responsible for replication of cells forming from the pond of the prebiotic earth by a statistical fluctuation. Also, of interest, is that Einstein in his explanation of Brownian motion made note of the fluctuation concept.

\section{Turbulence}

The possible connection between this phenomenon to a fluctuation-based origin is that they both share an abrupt structure change at some point - the distinct velocity for the onset of turbulent flow in the above case the and nucleation of a new phase in the latter. For fluid flow the change occurs at large values of the Reynold's number; "R" defined in equation 1. [10]

$$
\mathrm{R}=\mathrm{LV} / \mu
$$

Here $\mathrm{L}$ is the pipe diameter, $\mathrm{V}$ the fluid velocity and $\mu$ the fluid viscosity. The Navier-Stokes equation (shown as equation 2) is predictive in the flow behavior of values of $\mathrm{R}<$ 1000 but some sort of nonobvious instability (turbulent flow) sets in as the velocity increases beyond some value $\mathrm{R}>5000$. One might be tempted to call this the "nucleation" of turbulence since there is a sudden onset threshold like phenomenon at a critical fluid velocity. This will be the common theme contained in this paper

Continuity equation

$$
\nabla \cdot \vec{V}=0
$$

Momentum Equations

$$
\rho \frac{D \vec{V}}{D t}=-\nabla \rho+\rho \vec{g}+\mu \nabla^{2} \vec{V}
$$

\section{Liesegang Effect}

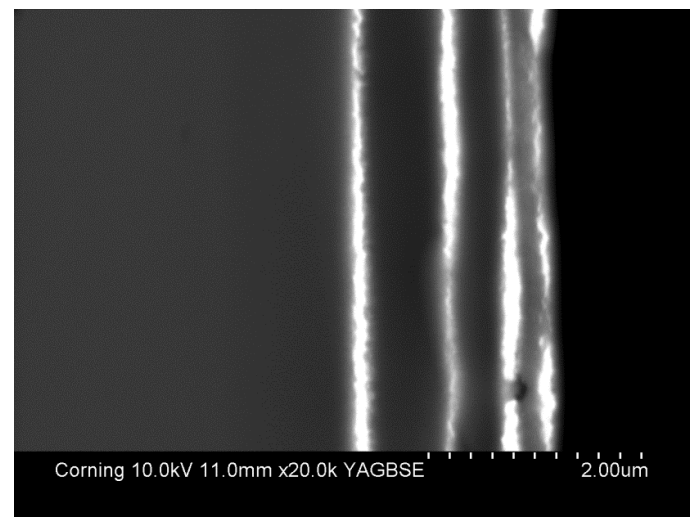

Figure 1. Electron micrograph of the Liesegang phenomenon; Ag ionexchanged into alkali containing glass the reduced in $\mathrm{H} 2400 \mathrm{C} 2 \mathrm{~h}$.

In this phenomenon a discrete layering of an Ag-metal phase spontaneously occurs in a glass that has undergone an $\mathrm{Ag}$ for alkali ion exchange then given post thermal treatment.
An example of the layering phenomenon is shown in Figure 1 for $\operatorname{Ag}(+)$ ion-exchanged glass when heated to a particular temperature and time in a hydrogen atmosphere. 5 The common description of this effect is in terms of a system where the nucleation of the phase is very slow with respect to sudden growth stage. However, this can be considered as an effect that goes through a critical stage where the layering begins to form. An interesting aspect of the layering behavior is that it involves counter diffusion of the Ag-ion. In other words, from an initial uniform ion exchange, represented by the mathematical $\operatorname{erfc}(z)$ profile, where the Ag-ion concentration it is deeper than the depth of the last layer.

Below are the equations 3 used to represent the process. The bold $\mathrm{H}$ is the Heaviside step function; A represents $\operatorname{Ag}(+1), \quad S$ is the reduces $\operatorname{Ag}(0)$ and $H$ represents the hydrogen concentration.

$$
\begin{aligned}
\frac{\partial}{\partial t} A(x, t) & =D_{A} \frac{\partial^{2}}{\partial x^{2}} A(x, t)-k_{1} A H \mathrm{H}\left(A H-K_{S}\right)-k_{2} A H S \\
\frac{\partial}{\partial t} H(x, t) & =D_{H} \frac{\partial^{2}}{\partial x^{2}} H(x, t)-k_{1} A H \mathrm{H}\left(A H-K_{S}\right)-k_{2} A H S \\
\frac{\partial}{\partial t} S(x, t) & =D_{S} \frac{\partial^{2}}{\partial x^{2}} S(x, t)-k_{1} A H \mathrm{H}\left(A H-K_{S}\right)-k_{2} A H S
\end{aligned}
$$

Equations 3 are essentially the classical Diffusion equation adding an abrupt threshold reaction term indicating the reduction of $\operatorname{Ag}(+)$ to $\operatorname{Ag}(0)$ stage. [11].

The condition of instability comes into play when the ratio $\mathrm{k} 2 / \mathrm{k} 1$ is large and the reaction rate constant $\mathrm{K}_{2}<\mathrm{AH}$; that is when the nucleation rate is very small relative to the $\operatorname{Ag}(0)$ growth. Here we have ad hoc added an abrupt nucleation term in the chemical reaction term using the Heaviside function. This means we have simulated what we propose happens spontaneously from concentration fluctuations as we will discuss in section IV. This additional term results in the computer simulation solution of the above equation which shows the onset of the instability and the formation of the layers thus verifying the result of such a model. This supports (as we discuss) our conjecture that the inclusion of a step-like function leads to sudden critical point similar to turbulence and nucleation in general.

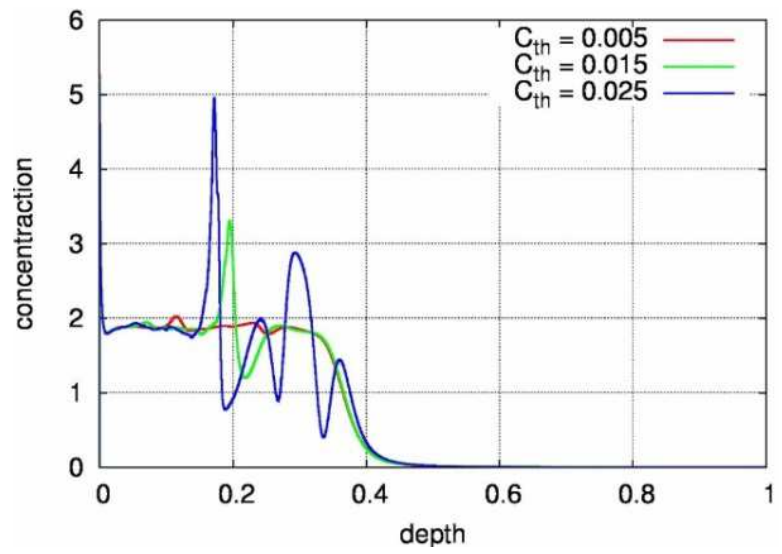

Figure 2. Computer simulation of equation $3\left(C_{t h}=K_{2}\right)$. 


\section{Theoretical Considerations}

Although the specific equations for the Liesegang and Navier-Stokes are quite different they nevertheless both contain time and spatial partial differential derivatives which can create complex responses of the respective quantities velocity in the turbulence example and concentration in the Liesegang case. Somehow, with this mathematical complexity, the instability arises [12], although, not explicitly known how. However, in the Liesegang formulation of equation 3 we have inserted "a priori" into the diffusion equation - a step like function and the layering property is exhibited. Because of this the comparison, the sudden onset of turbulence seems reasonable and they can in both cases be related to nucleation like phenomenon.

\section{Fluctuation and Dissipation}

We will be proposing the approach here that the nucleation phenomenon originates from the ongoing local ubiquitous dynamical structural changes in the material (aka, noise). The key noise parameter here is measured as the mean square dimension of these fluctuations. [8] This will be used as a measure as the "effective extent" of the nucleating region as shown in the expression shown here as equation 4. By fluctuation in this case represents any spontaneous structural change of a given spatial size as given in equation 4 .

Consider the Einstein definition of the mean square position for Brownian motion

$$
<x^{2}>=\int_{-\infty}^{\infty} P(x, t) x^{2} d x=2 D t
$$

probability function is

$$
P(x, t)=\left(\frac{1}{4 \pi D t}\right) \exp \left(-\frac{x^{2}}{4 D t}\right)
$$

Here " $\mathrm{D}$ " is the diffusion as the following (Einstein's theory)

$$
D=k_{B} T / 6 \pi \mu a
$$

Here $\mu$ is the viscosity and "a" is a particle size therefor the expression for the mean square fluctuation is the folllowing.

$$
<\mathrm{x}^{2}>=2 \mathrm{k}_{\mathrm{B}} \mathrm{Tt} / 6 \mu \mathrm{a}
$$

The issustructuree here is to define the what nucleating defect is diffusing in a glass. Suppose one assumes that the" particle size" in equation 7 is some sort of mobile defect in the glass network and imagine its mean square position can be thought as a measure of its spatial extent in the glass structure which diffuses. This could be a composition fluctuation as mentioned above [3] caused by a foreign ion. More likely candidiates are network disruptions a "strained bond" for example [13]. These are Si-O rings with less that 6 $\mathrm{Si}$ atoms. Also in alumino and boro-silicates there can also be local Al-O-Si and B-O bonding that could well be the fluctuation species that diffuse through the network and define the diffusion. The spatial extent of these structural disruption would define $\left\langle\mathrm{x}^{2}\right\rangle$ in equation 7 . One could conjecture that the viscosity term could also be involve the frequency of a distinct vibrational mode whose frequency is strongly temperature dependent as expressed in equation 8;

$$
<\mathrm{x}^{2}>\sim \mathrm{kT} / \grave{\omega}
$$

In view of the above analogies, temperature in the classical nucleation case would now be equivalent to velocity in the fluid flow case. That is, in the fluid flow case the instability occurs as one increases the velocity whereas in the classical nucleation situation the rate is increased with temperature. For the Liesegang effect it is the Ag-ion diffusion

In the normal thermal schedule that is used for glassceramics [2] the nucleation temperature is taken somewhat above the so-called transition temperature $\mathrm{Tg}$ which experimentally is approximated by the temperature at which there is a distinct break in the viscosity vs. temperature curve. There is no universal agreement or theoretical basis for this break other than it must represent a structural change of some kind and would be the temperature where the mean square value beomes critical.

\section{Conclusions}

The above approach has been proposed try to connect or relate the three apparently disparate phenomena as all varieties from the same nucleation point of view. We further suggest that the appropriate way to address the phenomena is using a fluctuation theory approach where all three of the phenomena we have discussed have their origin from random fluctuations.

In the case of the Liesegang effect where the instability shown in Figure 2 is intended to represent the nucleation event. By virtue of the proposed similarity this would be true in some way for all three phenomena. A specific representation of the common origin of proposed instability is indicated with the insertion of a term in equation 3 that was intended to simulate nucleation the shold.

\section{References}

[1] F. J. Dyson "Infinite in all directions" First Perennial, addition 1988 (Harper Collins).

[2] G. Wolfram and BH. Beall, Glass-Ceramic Technolgy. Am. Cer. Soc. 2019.

[3] Marie Guignard et al Structural fluctuations and role of Ti as nucleating agent, J. Non-crystal Solids v 356, 368 (2010).

[4] F. W, McMillan Glass Ceramics, Academic Press 1979.

[5] S. D, Stookey US patent 292097`A 1956

[6] E. D. Zanoto and V. M Fokin, Phil. Teans. R. Soc. Londonv 361, 591, (2003).

[7] N. F. Borrelli Photosensitive Glasses and Glass-ceramics CRC press 2016. 
[8] D. Landau, Statistical Phys. Butterworth-Wiley 3rd ed, 1980.

[9] P. W. Milonni, “An Introduction to Quantum Optics and Quantum Fluctuations” Oxford Press, 2019.

[10] J. Von Neumann, Collected Works Volume 6, Pergamon Press 1963.
[11] N. F. Borrelli et al; private unpublished data.

[12] Turing A. M. Phil. Trans. Royal Soc. London, 237(641) 37 (1952).

[13] K. Awazu "Strained bonds in silica" J. Appl. Phys. 94 (10) $6243(2003)$. 